\title{
Marginal deformations in string field theory
}

\section{Ehud Fuchs and Michael Kroyter}

Max-Planck-Institut für Gravitationsphysik, Albert-Einstein-Institut

14476 Golm, Germany

E-mail: udif@aei.mpg.de, mikroyt@aei.mpg.de

\section{Robertus Potting}

CENTRA, Departamento de Física, Faculdade de Ciências e Tecnologia, Universidade do Algarve, 8005-139 Faro, Portugal

E-mail: rpotting@ualg.pt

ABSTRACT: We describe a method for obtaining analytic solutions corresponding to exact marginal deformations in open bosonic string field theory. For the photon marginal deformation we have an explicit analytic solution to all orders. Our construction is based on a pure gauge solution where the gauge field is not in the Hilbert space. We show that the solution itself is nevertheless perfectly regular. We study its gauge transformations and calculate some coefficients explicitly. Finally, we discuss how our method can be implemented for other marginal deformations.

KEYwords: Gauge Symmetry, String Field Theory, Bosonic Strings. 


\section{Contents}

1. Introduction 1

2. The photon marginal deformation 3

3. Gauge-choice independence 77

3.1 Ignoring counter terms

3.2 Including counter terms 10

4. Evaluating coefficients in the oscillator representation 14

5. Other marginal deformations

6. Comparing with former solutions 18

7. Conclusions 19

A. Proving the equivalence of $(6.4)$ and $(6.5) \quad 20$

\section{Introduction}

The recent analytic construction by Schnabl [1] (see also [2-11]) of an exact solution in classical open string field theory (OSFT) [12] corresponding to the tachyon vacuum has given a renewed impetus in using OSFT as a tool in the analysis of open string vacua. In particular, it allowed for a proof of the first two of Sen's conjectures [13, 14. A class of related solutions based on general projectors has been developed in 15.

This advance raised the hope that other solutions can also be found, such as lump solutions and marginal deformations. That marginal deformations can be described within the framework of string field theory was shown by Sen [16-18]. There, it was shown that boundary marginal deformations can be described within OSFT, while bulk marginal deformations can be described using a non-polynomial closed string field theory, such as 19]. These solutions were first investigated using level truncation in the Siegel gauge [20]. Other studies of these solutions appeared in [21-29].

More recently, a recursive procedure has been developed by using the techniques employed in Schnabl's solution (in particular, the $\mathcal{B}_{0}$ gauge), yielding exactly marginal deformations order by order in a parameter $\lambda$ parameterizing the exactly flat direction [30, 31]. This approach was generalized [32, 33] to describe also the first analytical solutions of superstring field theory [34]. 
The approach of [30, 31] gives an explicit solution for marginal deformation generated by current operators which have a regular OPE with themselves. For the more interesting case, such as the photon marginal deformation, where the OPE of the vertex operator $V(z)$ defining the marginal deformation with itself is $V(0) V(z) \sim 1 / z^{2}$, divergences arise as the separations of the boundary insertions used in constructing the solution go to zero. This makes it necessary to add counter terms in order to cancel these divergences. However, the form of these counter terms is known only up to the third order and it is not a priori clear that counter terms for higher orders exist.

In this work we propose an alternative approach toward the analytical construction of exactly marginal deformations. It is based on solutions that are formally pure gauge, but nonetheless nontrivial as the gauge parameter we employ is not in the physical Hilbert space. As we will show, this method allows us to obtain an explicitly defined solution, perturbative in the above-mentioned parameter $\lambda$. As the insertions of the vertex operator in our approach remain at a finite distance, the divergences encountered in [30, 31] do not arise. However, it turns out that the solutions we obtain at first instance are singular in the sense that there is a non-normalizable dependence on the center of mass coordinate $x_{0}$. Only with a carefully chosen set of counter terms is it possible to regularize this unwanted dependence on $x_{0}$, such that the solutions are in fact independent of the center of mass coordinate.

Our approach starts off with the pure gauge solutions for string field theory of the form [2, 6]

$$
\Psi=(1-\lambda \phi) Q \frac{1}{1-\lambda \phi}=Q \phi \frac{\lambda}{1-\lambda \phi},
$$

which have the structure of a pure gauge solution generated by the gauge field

$$
\Lambda=-\log (1-\lambda \phi) .
$$

This is the case, because the finite gauge transformation in string field theory takes the form

$$
\Psi \rightarrow e^{-\Lambda}(\Psi+Q) e^{\Lambda}
$$

and we look for a gauge equivalent of the trivial solution $\Psi=0$.

It seems that this procedure cannot generate any non-trivial solutions. However, this solution can become a physical one in several ways. The first option is to have a finite radius of convergence with respect to $\lambda$. By a rescaling of $\phi$ this value $\lambda_{\text {crit }}$ can be set to unity. Then, for $|\lambda|<1$ the solution is indeed a gauge solution, while for $|\lambda|>1$ it is not well defined. For $\lambda= \pm 1$ the solution can either be gauge solution, not be well defined, or be a physical solution. Indeed, Schnabl's solution for the tachyon vacuum [1] is just of this form. There, the solution at $\lambda=-1$ is not well defined, while the $\lambda=1$ case is the desired solution (after proper regularization). A variant of this method would be to have $\lambda_{\text {crit }}=\infty$ such that the solution is well defined, but non-gauge at least in one of the limits $\lambda \rightarrow \pm \infty$. Another option would be to have some sort of a singular $\phi$, such that the solution itself is regular. Then, $Q \phi$ is an exact solution in a "large Hilbert space", but is a nontrivial element of the cohomology when considering the smaller Hilbert space. This is the method that we want to employ. 
The rest of the paper is organized as follows. In section 2 we introduce the analytical solution describing the photon marginal deformation $\lambda c \partial X$. Then, in section 3, we discuss other similar solutions and the issue of gauge equivalence. Our solution is obtained using CFT methods. Therefore, we devote section 1 to the oscillator form of the solution. Our construction is especially useful for solutions whose OPE is singular, such as the photon marginal deformation. However, it can be used also to describe other marginal deformation. This issue is studied in section 5. Next, in section 6 we comment on the relation of our solution to the previously found ones. Finally, we present our conclusions in section 0 .

Following are some of the conventions we use in the paper. Schnabl's solution is based on the wedge states [35-38]

$$
\psi_{n}=|n+1\rangle=\hat{U}_{n+1}|0\rangle=e^{\frac{1-n}{2} \hat{\mathcal{L}}_{0}}|0\rangle,
$$

where we use the notations

$$
\hat{U}_{n} \equiv U_{n}^{\dagger} U_{n}, \quad \hat{\mathcal{L}}_{0} \equiv \mathcal{L}_{0}+\mathcal{L}_{0}^{\dagger} .
$$

Here, $\mathcal{L}_{0}$ is the zero mode of the energy momentum tensor in the coordinates

$$
z=\tan ^{-1} \xi
$$

and $\mathcal{L}_{0}^{\dagger}$ is its conjugate. The $\xi$ coordinate is the standard one, where the local coordinate patch is half a unit circle. The $z$ coordinate system is natural when working with insertions over the wedge state $|2\rangle$, which is the $\mathrm{SL}(2)$ invariant vacuum. A natural generalization is to the coordinate system of the wedge state $|n\rangle$

$$
z^{(n)}=\frac{n}{2} z .
$$

Throughout the paper we work in the $z^{(n)}$ coordinates, when considering states built as insertions over the wedge state $|n\rangle$. All operators, such as $\partial X, c, \ldots$ should be understood as defined in the relevant coordinate system. Also, throughout the paper we star-multiply string fields, keeping the star product implicit. Similarly, a function of a string field represents the Taylor expansion of the function, with products given by star products.

\section{The photon marginal deformation}

Every marginal solution has a free parameter, which we mark as $\lambda$. It is natural to expand the solution in orders of $\lambda$,

$$
\Psi=\sum_{n=1}^{\infty} \lambda^{n} \psi_{n} .
$$

Plugging this into the equation of motion and comparing terms with the same power of $\lambda$ gives the recursive relations

$$
Q \psi_{n}=-\sum_{k=1}^{n-1} \psi_{k} \psi_{n-k} .
$$


For (formally) pure-gauge solutions (1.1) we get

$$
\psi_{n}=(Q \phi) \phi^{n-1}
$$

We will attempt to generate the photon marginal solution by making a singular choice of $\phi$

$$
\phi=\epsilon_{\mu} X^{\mu}(0)|0\rangle=\epsilon_{\mu} x_{0}^{\mu}|0\rangle \quad \Rightarrow \quad \psi_{1}=-i \sqrt{2} \epsilon_{\mu} \alpha_{-1}^{\mu} c_{1}|0\rangle .
$$

Here, we work in the $\alpha^{\prime}=1$ convention. Moreover, we are interested in boundary operators. Thus, ${ }^{1}$

$$
\partial X(z)_{\text {boundary }}=2 \partial X(z)_{\text {bulk }}
$$

In particular we have

$$
\partial X(0)|0\rangle=-i \sqrt{2} \alpha_{-1}|0\rangle
$$

The description of $\psi_{1}$ as a formal exact state (2.4) does not contradict the fact that $\psi_{1}$ is a physical state, i.e. non-exact. The reason is that $\phi$ is not in the Hilbert space, since a state whose representation in position space is

$$
\phi\left(x_{0}\right)=\left\langle x_{0} \mid \phi\right\rangle=x_{0},
$$

is not a normalizable state. It is therefore clear that our choice of $\phi$ generates a non-trivial solution. Still, to show that this solution is legitimate we have to show that all the $\psi_{n}$ 's are regular, i.e. $x_{0}$ independent. ${ }^{2}$ The first order, $\psi_{1}$ is regular by construction. The higher orders, however, have factors of $\phi^{n-1}$ in their definition (1.1) and are therefore singular, with $\mathcal{O}\left(x_{0}^{n-1}\right)|0\rangle$ terms. To correct these singularities we have to add counter terms to $\phi$, i.e. terms of the form

$$
\phi_{n} \approx X^{n},
$$

where we need to specify the location of each $X$ insertion. The gauge parameter $\phi$ becomes itself $\lambda$ dependent

$$
\phi=\sum_{n=1}^{\infty} \lambda^{n-1} \phi_{n} .
$$

For expressions of the form (2.8) to make sense, they should be normal ordered. Henceforth, normal ordering of operator insertions at the same point is implicit. The relevant normal ordering scheme is boundary normal ordering. In particular, all the variables that we use are defined only on the boundary.

To calculate these counter terms we need the commutation relation of $Q$ with $X(z)^{n}$ We use the relation

$$
\left[Q, e^{i p X(z)}\right]=\left(p^{2} \partial c e^{i p X}+i p c \partial X e^{i p X}\right)(z)
$$

\footnotetext{
${ }^{1}$ We shall henceforth omit the polarization vector $\epsilon_{\mu}$ and the $\mu$ index over $X$ for simplicity.

${ }^{2}$ An alternative procedure, which we don't pursue in this work, would be the construction of solutions with nontrivial but normalizable $x_{0}$ dependence. We believe that this line of thought may be of help in the construction of lump solutions.
} 
and derive it $n$ times to get

$$
\begin{aligned}
{\left[Q, X^{n}\right] } & =\left.\left(-i \partial_{p}\right)^{n}\left[Q, e^{i p X}\right]\right|_{p=0} \\
& =(-i)^{n}\left(2 i^{n-2}\left(\begin{array}{c}
n \\
2
\end{array}\right) \partial c X^{n-2}+i(i)^{n-1}\left(\begin{array}{c}
n \\
1
\end{array}\right) c \partial X X^{n-1}\right) \\
& =n c \partial X X^{n-1}-n(n-1) \partial c X^{n-2}
\end{aligned}
$$

Had we used (2.3) to construct the solution we would get

$$
\psi_{2}=\left(Q \phi_{1}\right) \phi_{1}=\hat{U}_{3} c \partial X\left(-\frac{\pi}{4}\right) X\left(\frac{\pi}{4}\right)|0\rangle .
$$

This is obviously singular, in the sense that the coefficient of $x_{0}$ is non-zero. To remedy this problem we add to $\phi$ a counter term of the form

$$
\phi_{2}=-\frac{1}{2} \hat{U}_{3} X^{2}\left(-\frac{\pi}{4}\right)|0\rangle .
$$

This gives a regular solution

$$
\begin{aligned}
\psi_{2} & =\left(Q \phi_{1}\right) \phi_{1}+Q \phi_{2} \\
& =\hat{U}_{3}\left(c \partial X\left(-\frac{\pi}{4}\right)\left(X\left(\frac{\pi}{4}\right)-X\left(-\frac{\pi}{4}\right)\right)+\partial c\left(-\frac{\pi}{4}\right)\right)|0\rangle \\
& \equiv(c \partial X, X)-(c \partial X X, 1)+(\partial c, 1) \\
& =(c \partial X, 1)((1, X)-(X, 1))+(\partial c, 1) .
\end{aligned}
$$

Here, a new notation has been introduced. As we consider here the wedge state $|3\rangle$ (formed by acting with $\hat{U}_{3}$ on the vacuum) with two possible insertion sites, we specify the insertions in these sites in a vector form, where the 1 stands for the identity insertion, i.e., no insertion. The multiplication in the last equality acts point wise.

At order $n$ we shall have to deal with $n$ insertions over the wedge state $|n+1\rangle$, symmetrically distributed around $z^{(n+1)}=0$, with a distance of $\frac{\pi}{2}$ between two consecutive insertion sites. There, we use an $n$-vector notation. Since the wedge state number is correlated with the number of insertion sites this notation is unambiguous.

The expression for $\psi_{2}$ seems regular since there is no $x_{0}$ dependence in $(1, X)-(X, 1)$, but this analysis is a bit too naive. One has to remember that we have normal ordering only for operators at the same site. Therefore,

$$
(c \partial X, 1)((1, X)-(X, 1))=: c \partial X\left(-\frac{\pi}{4}\right):: X\left(\frac{\pi}{4}\right):|0\rangle-: c \partial X X\left(-\frac{\pi}{4}\right):|0\rangle .
$$

It is easy to normal order this term and see that there is no $x_{0}$ dependence. This case is trivial since the new term that appears in the normal ordering has no $X$ dependence. For higher order terms, normal ordering $X^{n}$ will introduce $X^{n-2}$ and lower order operators. However, let $P\left(X_{1}, \ldots, X_{n}\right)$ be a polynomial in $X_{i}$, such that in each monomial operators at different sites are ordered from left to right. If $P$ is $x_{0}$ independent,

$$
\partial_{x_{0}} P=\sum_{k=1}^{n} \partial_{X_{k}} P=0,
$$


then the normal ordered expression given by

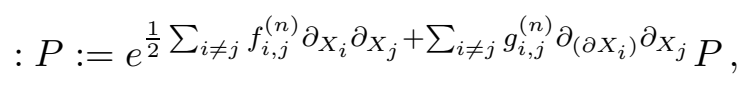

is also $x_{0}$ independent. This stems from the fact that the normal ordering operator inside the exponent commutes with the operator $\sum_{k=1}^{n} \partial_{X_{k}}$. This result is independent of the form of the structure functions $f_{i, j}^{(n)}, g_{i, j}^{(n)}$, as long as they are $X_{i}$-independent. ${ }^{3}$ Notice that the fully normal ordered polynomial $: P$ : can be manipulated as a standard polynomial, since all of its components commute with each other.

We now continue with the third order,

$$
\begin{aligned}
\psi_{3} & =\left(Q \phi_{1}\right) \phi_{1} \phi_{1}+\left(Q \phi_{2}\right) \phi_{1}+\left(Q \phi_{1}\right) \phi_{2}+Q \phi_{3} \\
& =(c \partial X, 1,1)\left((1, X, X)-(X, 1, X)-\frac{1}{2}\left(1, X^{2}, 1\right)\right)+(\partial c, 1, X)+Q \phi_{3} .
\end{aligned}
$$

The counter term $\phi_{3}$ is obviously needed. There is an $X(0)$ insertion multiplying $\partial c\left(-\frac{\pi}{2}\right)$, and the coefficient of the $(c \partial X)\left(-\frac{\pi}{2}\right)$ insertion also has an $x_{0}^{2}$ dependence. However, by setting

$$
\phi_{3}=\frac{1}{6} \hat{U}_{4} X^{3}\left(-\frac{\pi}{2}\right)|0\rangle=\frac{1}{6}\left(X^{3}, 1,1\right),
$$

we are led to

$$
\begin{aligned}
\psi_{3}= & Q \phi_{1} \phi_{1} \phi_{1}+Q \phi_{2} \phi_{1}+Q \phi_{1} \phi_{2}+Q \phi_{3} \\
= & (c \partial X, 1,1)\left((1, X, X)-(X, 1, X)-\frac{1}{2}\left(1, X^{2}, 1\right)+\frac{1}{2}\left(X^{2}, 1,1\right)\right) \\
& +(\partial c, 1,1)((1,1, X)-(X, 1,1)) .
\end{aligned}
$$

The coefficient of $\partial c$ is obviously regular and it is also clear that there is no $x_{0}^{2}$ term in the coefficient of $c \partial X$. There could have been a linear $x_{0}$ term there, though. In order to see that it is absent, we write $X$ at site $k$, as $x_{0}+\tilde{X}_{k}$, where $\tilde{X}_{k}$ is the regular part of the operator. Then, we see that

$$
\begin{aligned}
\left(x_{0}+\tilde{X}_{2}\right)\left(x_{0}+\tilde{X}_{3}\right)-\left(x_{0}+\tilde{X}_{3}\right)\left(x_{0}+\tilde{X}_{1}\right)+\frac{1}{2}( & \left.x_{0}+\tilde{X}_{1}\right)^{2}-\frac{1}{2}\left(x_{0}+\tilde{X}_{2}\right)^{2} \\
& =\tilde{X}_{2} \tilde{X}_{3}-\tilde{X}_{3} \tilde{X}_{1}+\frac{\tilde{X}_{1}^{2}}{2}-\frac{\tilde{X}_{2}^{2}}{2},
\end{aligned}
$$

so the linear term drops out as well, and the result is regular.

Continuing to higher orders, we guess that the general form of the counter terms is

$$
\phi_{n}=\frac{1}{n !}(-1)^{n-1}\left(X^{n}, 1, \ldots, 1\right)
$$

\footnotetext{
${ }^{3}$ While one can ignore the normal ordering issues for proving $x_{0}$-independence, they are relevant in some computations. The explicit structure functions will be needed for writing down the fully normal ordered solution.
} 
With this ansatz we have to check as before that all the $x_{0}$ coefficients vanish, without considering the issue of normal ordering. We prove this regularity condition by induction. Suppose that $\psi_{k}$ is regular for all $k<n$. The state $\psi_{n}$ is composed of all ways to partition the $n$ sites among the various $\phi_{k}$ 's, with $Q$ acting on the first site. We concentrate on the last $\phi$ in any given partition and write $\psi_{n}$ in terms of the $\psi_{k}$ 's as

$$
\psi_{n}=Q \phi_{n}+\sum_{k=1}^{n-1}\left(\psi_{n-k}, \phi_{k}\right) .
$$

We introduced a new notation here of composing two vectors into one longer vector. This is exactly the operation of the star product. Now,

$$
\partial_{x_{0}} \psi_{n}=Q \partial_{x_{0}} \phi_{n}+\sum_{k=1}^{n-1}\left(\left(\partial_{x_{0}} \psi_{n-k}, \phi_{k}\right)+\left(\psi_{n-k}, \partial_{x_{0}} \phi_{k}\right)\right) .
$$

The first term inside the sum drops out according to the induction hypothesis, while from (2.22) we see that

$$
\partial_{x_{0}} \phi_{k}=-\left(\phi_{k-1}, 1\right)
$$

Thus, we are left with

$$
\begin{aligned}
\partial_{x_{0}} \psi_{n} & =-\left(Q\left(\phi_{n-1}, 1\right)-\left(\psi_{n-1}, 1\right)+\sum_{k=2}^{n-1}\left(\psi_{n-k}, \phi_{k-1}, 1\right)\right) \\
& =-\left(\left(\psi_{n-1}, 1\right)-\left(\psi_{n-1}, 1\right)\right)=0
\end{aligned}
$$

where in the first equality we separated the $k=1$ term and in the second equality composed the first term with the sum as in (2.23). This completes the proof.

\section{Gauge-choice independence}

In this section we generalize our construction and find a large family of solutions. All these solutions should be gauge equivalent. Thus, we study the gauge equivalence of these solutions. First, in subsection 3.1 we consider gauge freedom ignoring counter terms. There, $\phi$ represents a given formal gauge generator of a specific marginal deformation, not necessarily the photon. As already mentioned, this construction fails to produce well defined solutions without introducing counter terms. In subsection 3.2 we study the consequences of adding them for the photon marginal deformation.

\subsection{Ignoring counter terms}

It is clear that the linearized equation of motion $\psi_{1}=Q \phi$ does not uniquely define the solution to be of the form (1.1), in which the $Q$ operates only on the leftmost $\phi$ at each order (2.3). For example, we could have considered instead

$$
\Psi_{R}=\frac{\lambda}{1+\lambda \phi} Q \phi,
$$


which is generated by the gauge field

$$
\Lambda=\log (1+\lambda \phi) .
$$

Here, $Q$ acts only on the rightmost $\phi$.

Do these solutions describe the same physical state? When several marginal directions exist, the non-linear (that is, finite) extension of the initial infinitesimal deformation is not unique, and can "point" in various (possibly $\lambda$-dependent) directions in the vector space of infinitesimal marginal deformations. However, it seems that in our construction the only marginal deformation considered is related to the zero mode of a single $X$ coordinate. Thus, all the solutions should be gauge equivalent. Indeed, at the second order, the difference between the two solutions is

$$
\psi_{2}^{L}-\psi_{2}^{R}=Q\left(\phi^{2}\right) .
$$

So they are indeed gauge equivalent to this order.

One can also consider other extensions of the solution. If we restrict ourselves to solutions, which depend only on $\phi$ and have a total $n^{\text {th }}$ power of $\phi$ at order $n$, then a general ansatz can be written as

$$
\Psi=\sum_{n=1}^{\infty} \lambda^{n} \sum_{k=1}^{n} \gamma_{n, k} \phi^{k-1}(Q \phi) \phi^{n-k},
$$

and the requirement that $\Psi$ is a solution imposes restrictions on the values of the coefficients $\gamma_{n, k}$. The general solution for $\gamma_{n, k}$ amounts to the freedom of adding $Q\left(\phi^{n}\right)$ at order $n$ to the solution. This is a straightforward generalization of (3.3), with one gauge parameter at each order.

Another way to represent the gauge freedom is by specifying $\Lambda$. Any $\Lambda$ that agrees to first order with the original one and depends only on powers of $\phi$ should be equivalent to it. Such a $\Lambda$ has a Taylor expansion

$$
\Lambda=\lambda \phi+\sum_{n=2}^{\infty} a_{n}(\lambda \phi)^{n}, \quad e^{\Lambda}=1+\lambda \phi+\sum_{n=2}^{\infty} b_{n}(\lambda \phi)^{n},
$$

with obvious relations between the coefficients $a_{n}, b_{n}$. Two solutions which agree up to order $n-1$, differ by

$e^{-\Lambda_{1}} Q e^{\Lambda_{1}}-e^{-\Lambda_{2}} Q e^{\Lambda_{2}}=\lambda^{n}\left(a_{n}^{(1)}-a_{n}^{(2)}\right) Q\left(\phi^{n}\right)+\mathcal{O}\left(\lambda^{n+1}\right)=\lambda^{n}\left(b_{n}^{(1)}-b_{n}^{(2)}\right) Q\left(\phi^{n}\right)+\mathcal{O}\left(\lambda^{n+1}\right)$.

Thus, we see that choosing the expansion coefficients $a_{n}$ or $b_{n}$ is equivalent to choosing $\gamma_{n, k}$ consistently. In particular it is easy to see that

$$
b_{n}=\gamma_{n, 1} .
$$

The two simple solutions described above correspond to

$$
\gamma_{n, k}^{L}=\delta_{k, 1}, \quad \gamma_{n, k}^{R}=(-1)^{n-1} \delta_{k, n} .
$$


The gauge freedom described above allows for a more symmetric solutions, such as

$$
\gamma_{n, k}^{S}=\frac{1}{2^{n-1}}(-1)^{k-1},
$$

which is generated by

$$
\Lambda=\log \left(\frac{1+\frac{\lambda \phi}{2}}{1-\frac{\lambda \phi}{2}}\right)=2 \tanh ^{-1}\left(\frac{\lambda \phi}{2}\right) .
$$

Another, relatively symmetric combination that we found is

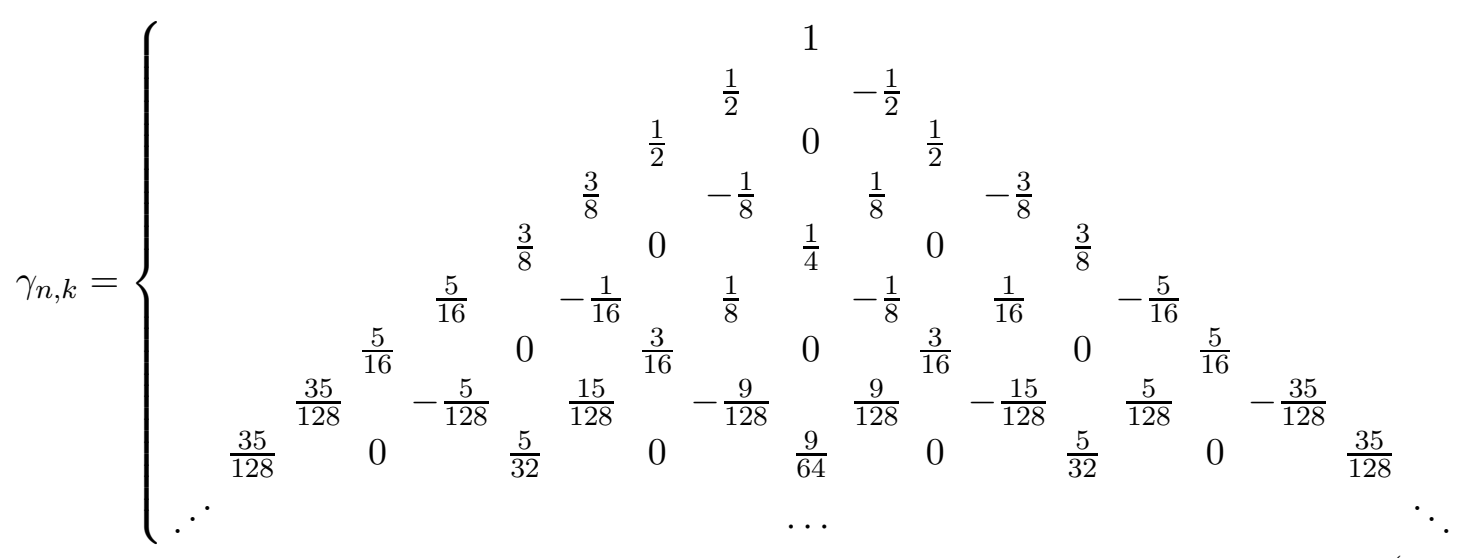

This expansion is generated by

$$
\Lambda=\frac{1}{2} \log \left(\frac{1+\lambda \phi}{1-\lambda \phi}\right)=\tanh ^{-1}(\lambda \phi),
$$

and the expansion coefficients have the peculiar property

$$
\sum_{k=1}^{n} \gamma_{n, k}=\left\{\begin{array}{ll}
0 & n \equiv_{2} 0 \\
1 & n \equiv_{2} 1
\end{array} .\right.
$$

With the richness of gauge descriptions one may wonder whether they all have the same range of validity in parameter space. Here, we have a single parameter $\lambda$, and it is clear on physical grounds that its value can be arbitrary. However, since we should not expect a gauge choice to be globally valid, it is possible that as we increase $\lambda$ the boundary of validity is attained for some of the gauge choices. There may be a subtlety here, since there can be a non-trivial relation

$$
\lambda_{\mathrm{SFT}}=f\left(\lambda_{\mathrm{CFT}}\right)=\lambda_{\mathrm{CFT}}+\ldots,
$$

with $f$ a monotonic function. It can happen that as $\lambda_{\mathrm{CFT}}$ goes to infinity, $\lambda_{\mathrm{SFT}}$ reaches a finite value. If this is the case, it can happen that the radius of convergence with respect to $\lambda_{\mathrm{SFT}}$ would always be finite. Also, note that the function $f$ generally depends on the solution. We saw that for our ansatz this reparametrization is absent for the leading order (3.6), but it can appear at higher ones. We assume for now that this does not happen.

As all the gauge choices that we explicitly considered so far are described by functions of $\lambda \phi$ with a finite radius of convergence, it seems plausible that the gauge choice would 
break down at some stage. ${ }^{4}$ One may hope that a wider range of validity is attained by choosing a function $\Lambda$ such that $e^{ \pm \Lambda}$ are complete. The simplest example of the form (3.6) is

$$
\Lambda=\lambda \phi
$$

With this choice one gets

$$
\gamma_{n, k}=\frac{(-1)^{k-1}}{n(k-1) !(n-k) !} .
$$

These coefficients have the peculiar property that

$$
\forall n>1 \quad \sum_{k=1}^{n} \gamma_{n, k}=0 .
$$

\subsection{Including counter terms}

In the previous subsection we have been ignoring counter terms. Now, we want to address the question of the uniqueness of the solution including the counter terms for the case of the photon marginal deformation. Any two solutions that are the same up to a given order $\Psi_{1}-\Psi_{2}=\mathcal{O}\left(\lambda^{n}\right)$, are gauge equivalent at the next order, provided that their difference is $Q$-exact to this order, $\Psi_{1}-\Psi_{2}=\lambda^{n} Q(\Upsilon)+\mathcal{O}\left(\lambda^{n+1}\right)$, as in (3.6). Recall that $Q$ acting on a singular expression should not be considered as $Q$-exact, but only as $Q$-closed. In this subsection we study the gauge freedom at the leading order and find the full (all order) gauge transformation between $\Psi_{L}$ and $\Psi_{R}$.

With the counter terms taken into account, the two simple solutions $\Psi_{L}, \Psi_{R}$ defined by equations (1.1) and (3.1) are generated by

$$
\begin{aligned}
& \phi_{L}=(X)-\frac{\lambda}{2 !}\left(X^{2}, 1\right)+\frac{\lambda^{2}}{3 !}\left(X^{3}, 1,1\right)+\ldots+\frac{(-\lambda)^{n-1}}{n !}\left(X^{n}, 1, \ldots, 1\right)+\ldots, \\
& \phi_{R}=(X)+\frac{\lambda}{2 !}\left(1, X^{2}\right)+\frac{\lambda^{2}}{3 !}\left(1,1, X^{3}\right)+\ldots+\frac{\lambda^{n-1}}{n !}\left(1, \ldots, 1, X^{n}\right)+\ldots
\end{aligned}
$$

The difference between the solutions $\Psi_{L}-\Psi_{R}$, before counter terms are taken into account is given at the second order by (3.3)

$$
\Delta_{2}=Q\left(\phi^{2}\right)=Q(X, X)
$$

This is obviously $Q$ acting on a singular expression. However, with counter terms taken into account the difference is in fact

$$
\Delta_{2}=Q\left((X, X)-\frac{1}{2}\left(X^{2}, 1\right)-\frac{1}{2}\left(1, X^{2}\right)\right)
$$

which is $Q$ on a regular expression. So the solutions are gauge equivalent at this order. This is the most general regular expression at this order, which is of the form of our ansatz.

\footnotetext{
${ }^{4}$ One should keep in mind, though, that the relevant product is the star product. So the series expansion should break down when an eigenvalue in some relevant space with respect to this product attends a critical value.
} 
The most general third order difference, between solutions of the form we consider here, that agree up to the second order, is

$$
\begin{aligned}
\Delta_{3}= & Q \phi_{3} \\
\phi_{3}= & \alpha_{1,1,1}(X, X, X)+\alpha_{3,0,0}\left(X^{3}, 1,1\right)+\alpha_{0,3,0}\left(1, X^{3}, 1\right)+\alpha_{0,0,3}\left(1,1, X^{3}\right) \\
& +\alpha_{2,1,0}\left(X^{2}, X, 1\right)+\alpha_{2,0,1}\left(X^{2}, 1, X\right)+\alpha_{1,2,0}\left(X, X^{2}, 1\right)+\alpha_{0,2,1}\left(1, X^{2}, X\right) \\
& +\alpha_{1,0,2}\left(X, 1, X^{2}\right)+\alpha_{0,1,2}\left(1, X, X^{2}\right) .
\end{aligned}
$$

The ten parameters $\alpha_{i, j, k}$ should be chosen such that $\Delta_{3}$ is regular. To that end, we should require that the coefficients of $c \partial X x_{0}^{2}, c \partial X x_{0} \tilde{X}_{i}$ and $\partial c x_{0}$ in the three sites should be zero. Thus, there are totally $3+3 \times 3+3=15$ linear equations restricting the values of the $\alpha_{i, j, k}$ 's. Twelve homogeneous linear equations in ten variables are of course dependent. It turns out that the general solution forms a four-dimensional space. That we have here four free parameters, whereas only a single gauge parameter was advocated in (3.4), stems from the fact that we are dealing here with a more general ansatz due to the appearance of counter terms. Now, to verify that all the solutions are the same also at the third order, we have to check the form of $\phi_{3}$ in this four-dimensional space. It turns out that it is indeed regular.

In a similar way at order $n$ we consider

$$
\Delta_{n}=Q \phi_{n}, \quad \phi_{n}=\sum_{\substack{i_{1}, \ldots, i_{n}=0 \\ i_{1}+\ldots+i_{n}=n}}^{n} \alpha_{i_{1}, \ldots, i_{n}}\left(X^{i_{1}}, \ldots, X^{i_{n}}\right) .
$$

Here, the number of coefficients is

$$
\#_{\alpha_{\vec{i}}}=\left(\begin{array}{c}
2 n-1 \\
n
\end{array}\right)
$$

Let us note that there are restrictions coming from the expansion of $c \partial X P_{n-1}\left(\tilde{X}_{i}, x_{0}\right)$ and $\partial c P_{n-2}\left(\tilde{X}_{i}, x_{0}\right)$, in the $n$ sites, where $P_{n-1}$ and $P_{n-2}$ are homogeneous polynomials of degrees $n-1, n-2$ respectively. A rank- $k$ homogeneous polynomial with $m$ variables is composed of

$$
C_{k, m} \equiv\left(\begin{array}{c}
k+m-1 \\
k
\end{array}\right)
$$

monomials. The constraints we have amount to requiring that the two polynomials depend only on $n$ out of their $n+1$ variables. Thus, the total number of equations is

$$
\#_{\mathrm{eq}}=n\left(\left(C_{n-1, n+1}-C_{n-1, n}\right)+\left(C_{n-2, n+1}-C_{n-2, n}\right)\right)=(3 n-4)\left(\begin{array}{c}
2 n-3 \\
n-1
\end{array}\right) .
$$

For $n>3$ the number of equations keeps being larger than the number of variables, just as is the case for $n=3$. At the fourth order only 20 out of a total of 80 equations (in 35 variables) are independent. Thus, the space of allowed $\phi_{4}$ is $35-20=15$-dimensional. It turns out that when restricted to this space of solutions $\phi_{4}$ is regular. 
We want to prove this result for the general case, that is, we want to show, that given $\alpha_{\vec{i}}$ such that $Q \phi_{n}$ is regular, so is $\phi_{n}$ itself. In the expression

$$
Q \phi_{n}=\sum_{\substack{i_{1}, \ldots, i_{n}=0 \\ i_{1}+\ldots+i_{n}=n}}^{n} \alpha_{i_{1}, \ldots, i_{n}} \sum_{k=1}^{n}\left(X^{i_{1}}, \ldots, i_{k} c \partial X X^{i_{k}-1}-i_{k}\left(i_{k}-1\right) \partial c X^{i_{k}-2}, \ldots, X^{i_{n}}\right)
$$

we have to demand that the coefficient of each $c \partial X$ and each $\partial c$, is $x_{0}$ independent. We write the coefficient of $c \partial X$ at site $k$ as

$$
\xi_{k}=\partial_{X_{k}} \sum_{\substack{i_{1}, \ldots, i_{n}=0 \\ i_{1}+\ldots+i_{n}=n}}^{n} \alpha_{i_{1}, \ldots, i_{n}}\left(X^{i_{1}}, \ldots, X^{i_{n}}\right)=\partial_{X_{k}} \phi_{n}
$$

while that of $\partial c$ can be written as

$$
\zeta_{k}=-\partial_{X_{k}}^{2} \sum_{\substack{i_{1}, \ldots, i_{n}=0 \\ i_{1}+\ldots+i_{n}=n}}^{n} \alpha_{i_{1}, \ldots, i_{n}}\left(X^{i_{1}}, \ldots, X^{i_{n}}\right)=-\partial_{X_{k}}^{2} \phi_{n}
$$

It is clear that $\partial_{x_{0}} \xi_{k}=0$ implies $\partial_{x_{0}} \zeta_{k}=0$, so it is enough to consider the former and what we have to show is that

$$
\partial_{x_{0}} \xi_{k}=\sum_{i=1}^{n} \partial_{X_{i}} \xi_{k}=0 \quad \forall k \quad \Rightarrow \quad \partial_{x_{0}} \phi_{n}=\sum_{k=1}^{n} \xi_{k}=0 .
$$

Now, consider

$$
\begin{aligned}
0 & =\sum_{k=1}^{n} X_{k} \sum_{i=1}^{n} \partial_{X_{i}} \xi_{k} \\
& =\sum_{i=1}^{n} \partial_{X_{i}} \sum_{k=1}^{n} X_{k} \partial_{X_{k}} \phi_{n}-\sum_{k=1}^{n} \partial_{X_{k}} \phi_{n} \\
& =(n-1) \sum_{i=1}^{n} \partial_{X_{i}} \phi_{n} \\
& =(n-1) \partial_{x_{0}} \phi_{n},
\end{aligned}
$$

where we used Euler's homogeneous function theorem. We see that for $n>1$ regularity of the coefficients indeed implies that of $\phi_{n}$, so all the solutions are indeed gauge equivalent, at least up to the first order where they differ.

It is clear that any gauge field $\phi$ that is $x_{0}$ independent produces an $x_{0}$ independent $Q \phi$. We have just prove also the opposite direction. Thus, we can now characterize the $\phi$ space in this way. This enables us to calculate the dimension of this space, since for a homogeneous polynomial of degree $n$,

$$
\partial_{x_{0}} P\left(x_{k}\right)=\sum \partial_{X_{i}} P\left(x_{k}\right)=0 \Rightarrow P=\sum k_{i_{2}, \ldots, i_{n}}\left(x_{1}-x_{2}\right)^{i_{2}} \cdots\left(x_{1}-x_{n}\right)^{i_{n}},
$$

with $k_{i_{2}, \ldots, i_{n}}$ a set of coefficients. From (3.26), we see that

$$
\operatorname{dim}\left(\phi_{n}\right)=\left(\begin{array}{c}
2 n-2 \\
n
\end{array}\right)
$$


which agrees with the quoted results for $n<5$. Moreover, we see that the most general solution can be found from (2.25), while in this subsection we studied the homogeneous version of this equation.

One of the gauge degrees of freedom is related to a change in $\gamma_{n, k}$. This is generated by $Q\left(\phi^{n}\right)$, that is, by $(X, \ldots, X)$. When one wants to consider a change in the gauge within a choice of $\gamma_{n, k}$ (or $\Lambda$ ), one should constrain the gauge parameter to the orthogonal space to this direction, say, by setting the coefficient of $(X, \ldots, X)$ to zero. However, it is not clear to us in which sense the inner product that we use in the space of $\alpha_{\vec{i}}^{\prime}$ 's is canonical. Thus, this choice of "orthogonal direction" is somewhat arbitrary.

This brings us back to the discussion regarding the range of validity of the different gauge choices. It is hard to disentangle the choice of counter terms from the choice of $\gamma_{n, k}$. Moreover, with counter terms taken into account, the gauge parameter $\phi$ is itself $\lambda$ dependent. Thus, it is possible that the solutions $\Psi_{L, R}$ have an infinite radius of convergence. It can also happen that the solution (3.15) would have a finite radius of convergence with an appropriately chosen set of counter terms and the radius can also depend on the specific choice. The easiest way in practice to generate an appropriate set of counter terms for a given $\gamma_{n, k}$, is by imposing (2.25), while fixing the coefficients $\alpha_{1, \ldots, 1}$ that generate the specific choice of $\gamma_{n, k}$. We try to estimate the radius of convergence of the solution $\Psi_{L}$ in section 1 .

We now want to prove that the two solutions that we found explicitly $\Psi_{L}, \Psi_{R}$, which differ at the second order, are indeed exactly gauge equivalent. To that end we first note that (1.3) implies that when two finite gauge transformations are combined, the resulting transformation is given by

$$
e^{\Lambda}=e^{\Lambda_{1}} e^{\Lambda_{2}}
$$

We now combine the singular gauge transformations that send $\Psi_{L}$ to the trivial solution and the trivial solution to $\Psi_{R}$, to get the gauge transformation from $\Psi_{L}$ to $\Psi_{R}$,

$$
\begin{aligned}
e^{\Lambda} & =e^{-\Lambda_{L}} e^{\Lambda_{R}} \\
& =\left(1-\lambda \phi_{L}\right)\left(1+\lambda \phi_{R}\right) \\
& =\sum_{n=0}^{\infty} \frac{(-\lambda)^{n}}{n !} \sum_{m=0}^{\infty} \frac{\lambda^{m}}{m !} \underbrace{\left(X^{n}, 1, \ldots, 1, X^{m}\right)}_{n+m} \\
& =\sum_{n=0}^{\infty} \lambda^{n} \sum_{m=0}^{n} \frac{(-1)^{m}}{(n-m) ! m !} X_{1}^{m} X_{n}^{n-m} \\
& =\sum_{n=0}^{\infty} \frac{\lambda^{n}}{n !}\left(X_{n}-X_{1}\right)^{n},
\end{aligned}
$$

where in the second line we suppressed the irrelevant sites. However, one should remember that the expression at order $n$ is defined over the wedge state $|n\rangle$. We see that the resulting transformation is manifestly regular, so $\Psi_{L, R}$ are truly gauge equivalent to all orders. 


\section{Evaluating coefficients in the oscillator representation}

Next we want to calculate the coefficients of the different fields in the oscillatory expansion for the photon marginal solution. This requires normal ordering our expression.

The easiest way to work with the (non-primary) $X^{n}$ fields is by their defining expression

$$
\left.X^{n}(z) \equiv\left(-i \partial_{p}\right)^{n} e^{i p X(z)}\right|_{p=0} .
$$

These fields are normal ordered in the sense of the $z$ coordinates. To find the OPE of two $X\left(z^{(n)}\right)$ operators we recall first that in the $\xi$ plane

$$
\partial X\left(\xi_{1}\right) \partial X\left(\xi_{2}\right)=: \partial X\left(\xi_{1}\right) \partial X\left(\xi_{2}\right):-\frac{2}{\xi_{12}^{2}},
$$

where the factor of 2 comes from the fact that we are working with boundary operators as in (2.6). The conformal transformation

$$
z^{(n)}=\frac{n}{2} \tan ^{-1} \xi
$$

gives

$$
\partial X\left(z_{1}^{(n)}\right) \partial X\left(z_{2}^{(n)}\right)=: \partial X\left(z_{1}^{(n)}\right) \partial X\left(z_{2}^{(n)}\right):-\left(\frac{2}{n}\right)^{2} \frac{2}{\sin ^{2}\left(\frac{2}{n} z_{12}^{(n)}\right)} .
$$

Integrating we get

$$
\begin{aligned}
\partial X\left(z_{1}^{(n)}\right) X\left(z_{2}^{(n)}\right) & =: \partial X\left(z_{1}^{(n)}\right) X\left(z_{2}^{(n)}\right):-\frac{4}{n} \cot \left(\frac{2}{n} z_{12}^{(n)}\right) \\
X\left(z_{1}^{(n)}\right) X\left(z_{2}^{(n)}\right) & =: X\left(z_{1}^{(n)}\right) X\left(z_{2}^{(n)}\right):-2 \log \left(\sin \left|\frac{2}{n} z_{12}^{(n)}\right|\right)
\end{aligned}
$$

Other than giving the form of the singular part of the OPE, these equations also define what we mean by normal ordering in the $z^{(n)}$-plane.

The $X^{m}$ operators are not primaries. We would need their transformation properties for going between the $z^{(n)}$ and the $\xi$ planes. From their definition, it follows that

$$
\begin{aligned}
X^{m}(\xi) & =\left.\left(-i \partial_{p}\right)^{m} e^{i p X(\xi)}\right|_{p=0} \\
& =\left.\left(-i \partial_{p}\right)^{m}\left(\left(\frac{n}{2} \cos ^{2}\left(z^{(n)}\right)\right)^{p^{2}} e^{i p X\left(z^{(n)}\right)}\right)\right|_{p=0} \\
& =\sum_{j=0}^{\left\lfloor\frac{m}{2}\right\rfloor} \frac{m !}{(m-2 j) ! j !} X^{m-2 j}\left(z^{(n)}\right) \log ^{j}\left(\frac{2}{n \cos ^{2}\left(z^{(n)}\right)}\right) \\
& =e^{-\log \left(\frac{n}{2} \cos ^{2}\left(z^{(n)}\right)\right) \partial_{X}^{2}} X^{m}\left(z^{(n)}\right),
\end{aligned}
$$

where in the second equality we used Leibnitz' formula, as well as the fact that only an even number of $p$-derivatives on $c^{p^{2}}$ gives a non-zero result at $p=0$. Hence, also the floor-function on the sum. Similarly,

$$
\begin{aligned}
X^{m}\left(z^{(n)}\right) & =\sum_{j=0}^{\left\lfloor\frac{m}{2}\right\rfloor} \frac{m !}{(m-2 j) ! j !} X^{m-2 j}(\xi) \log ^{j}\left(\frac{n}{2\left(1+\xi^{2}\right)}\right) \\
& =e^{-\log \left(\frac{2}{n}\left(1+\xi^{2}\right)\right) \partial_{X}^{2}} X^{m}(\xi) .
\end{aligned}
$$


Equations (4.7) and (4.8) relate powers of $X$ at the same point that are normal ordered in the sense of the $\xi$ coordinates to those that are normal ordered in the sense of the $z^{(n)}$ coordinates, and vice-versa (we note again that normal ordering is taken to be implicit for operators inserted at the same point). We can now use an argument analogous to the one following (2.17) to show that our construction, which leads to $x_{0}$-independence in the sense of the $z^{(n)}$ coordinates, automatically implies $x_{0}$-independence in the sense of the $\xi$ coordinates. Indeed, noting that

$$
\partial_{x_{0}} X^{m}=\partial_{X} X^{m}
$$

the fact that the final expression of (4.8) involves the exponent of an operator independent of $m$ commuting with $\partial_{X}$ shows that $x_{0}$-independent expressions constructed using the $z^{(n)}$ coordinates translate to $x_{0}$-independent expressions in the sense of the $\xi$ coordinates.

We also need the normal ordering coefficients for (2.17). In the $z^{(n)}$ coordinate they are

$$
f_{i, j}^{(n)}=-2 \log \left(\sin \left|\frac{2}{n} z_{i j}^{(n)}\right|\right), \quad g_{i, j}^{(n)}=-\frac{4}{n} \cot \left(\frac{2}{n} z_{i j}^{(n)}\right),
$$

while in the upper half plane

$$
f_{i, j}^{\mathrm{UHP}}=-2 \log \left|\xi_{i j}\right|, \quad g_{i, j}^{\mathrm{UHP}}=-\frac{2}{\xi_{i j}} .
$$

We want to calculate the coefficients of the lowest state, i.e. the tachyon state $c_{1}|0\rangle$, as well as that of the photon in the direction in which we induce the marginal deformation, i.e. the state $\alpha_{-1} c_{1}|0\rangle$. This corresponds to calculating the expectation values

$$
\begin{aligned}
T(\lambda) & =\sum_{n=1}^{\infty} \lambda^{n} T_{n}, & T_{n} & =\frac{2}{n+1}\left\langle\partial c c, \psi_{n}\right\rangle_{n+1}, \\
A(\lambda) & =\sum_{n=1}^{\infty} \lambda^{n} A_{n}, & A_{n} & =\frac{i}{\sqrt{2}}\left\langle\partial c c \partial X, \psi_{n}\right\rangle_{n+1},
\end{aligned}
$$

where the prefactor $\frac{n+1}{2}$ in the first equation comes from the conformal transformation to $C_{\frac{(n+1) \pi}{2}}$, the cylinder with circumference $\frac{(n+1) \pi}{2}$ and in the second expression we took into account (2.6). Symmetry dictates that for $T(\lambda)$ only the even powers are nontrivial, while for $A(\lambda)$ only the odd powers are non trivial.

For the ghost sector the only correlators that we need are

$$
\begin{gathered}
\langle\partial c c, c, \underbrace{1, \ldots, 1}_{n-2}\rangle=\frac{n^{2}}{4} \sin ^{2}\left(\frac{\pi}{n}\right), \\
\langle\partial c c, \partial c, \underbrace{1, \ldots, 1}_{n-2}\rangle=\frac{n}{2} \sin \left(\frac{2 \pi}{n}\right) .
\end{gathered}
$$

Here we introduced a new notation for the expectation value of a wedge state $n$ with the $n$ possible canonical insertion points. For the matter sector only the constants that arise 
from the normal ordering procedure as well as expressions that are a priori $X$ independent $^{5}$ can contribute.

The first coefficient is ${ }^{6}$

$$
A_{1}=\frac{i}{\sqrt{2}}\langle\partial c c, c\rangle\langle\partial X, \partial X\rangle=-i \sqrt{2}
$$

where we used (4.4). This result is trivial, since this is nothing but the initial condition for the solution (2.4). Next, we have

$$
T_{2}=\frac{2}{3}(\langle\partial c c, c, 1\rangle(\langle 1, \partial X, X\rangle-\langle 1, \partial X X, 1\rangle)+\langle\partial c c, \partial c, 1\rangle),
$$

where we substituted the result for $\psi_{2}$ (2.14). The second term does not contribute, since it contains only $X$ insertions at the same point, which are already normal ordered. The first term contributes the normal ordering constant and the third term is the unique case, where there is no $X$ dependence. All in all we get

$$
T_{2}=\frac{2}{3}\left(\frac{9}{4} \sin ^{2}\left(\frac{\pi}{3}\right)\left(-\frac{4}{3}\right) \cot \left(\frac{2}{3}\left(-\frac{\pi}{2}\right)\right)+\frac{3}{2} \sin \left(\frac{2 \pi}{3}\right)\right)=\sqrt{3} .
$$

Here, we used (4.5).

In a similar manner we can calculate higher order terms. The only limitation is that the number of terms increases very fast and the analytic expressions become extremely cumbersome. We calculated the following values numerically:

$$
\begin{aligned}
& T_{4}=1.39848, \quad T_{6}=0.863328, \quad T_{8}=0.477264, \\
& T_{10}=0.243907, \quad T_{12}=0.117105, \quad T_{14}=0.0534555, \\
& A_{3}=-5.2229 i, \quad A_{5}=-6.61142 i, \quad A_{7}=-5.84034 i, \\
& A_{9}=-4.23182 i, \quad A_{11}=-2.67936 i, \quad A_{13}=-1.53481 i \text {. }
\end{aligned}
$$

These numbers seem to indicate that we have a finite radius of convergence.

A different choice of counter terms would have produced different coefficients. It seems that the number of degrees of freedom in choosing the counter terms grows fast with the level. Thus, one may hope that it would be possible to choose them in a way that would minimize the coefficient at each level. One has to remember though, that there are also an infinity of other coefficients, that we did not evaluate. For the series to converge, we have to impose convergence on the whole infinite set, and a gauge that minimizes one coefficient can be less adequate for minimizing the others. This issue deserves further study.

Note, however, that the change of a gauge at level $n$ cannot modify the coefficient of $A_{n}$. This results from the closeness of the bra state used for extracting the photon coefficient. Let the gauge change be given in the leading order by $Q \phi_{n}$. The change it induces is

$$
\left\langle\partial c c \partial X, Q \phi_{n}\right\rangle=-\left\langle Q(\partial c c \partial X), \phi_{n}\right\rangle=0
$$

\footnotetext{
${ }^{5}$ The only such case is the operator $\partial c$ that arises at the second order.

${ }^{6}$ We work in conventions in which $\lambda$ is imaginary. Thus, odd coefficients appear to be imaginary.
} 
This, however, raises a puzzle, as we are using a similar form for our initial condition, $\psi_{1}=Q X(0)|0\rangle$. So it may seem that $A_{1}=0$, which is obviously wrong. This stems from the fact that $X|0\rangle$ lies outside the Hilbert space. The operator $x_{0}$ turns the usual infinite volume factor $\delta(p)$ into $\delta^{\prime}(p)$. This works as follows, we add momentum dependence to the bra state. Now,

$$
\begin{aligned}
\left\langle(\partial c c \partial X)\left(z_{1}\right) e^{i p X}\left(z_{2}\right), Q X\right\rangle & =-\left\langle(\partial c c \partial X)\left(z_{1}\right) Q\left(e^{i p X}\right)\left(z_{2}\right), X\right\rangle \\
& =-\left\langle(\partial c c \partial X)\left(z_{1}\right)\left(p^{2} \partial c e^{i p X}+i p c \partial X e^{i p X}\right)\left(z_{2}\right), X\right\rangle .
\end{aligned}
$$

Here, we use point split regularization due to the appearance of the $\partial X$ term. The $\partial X \partial X$ singularity is exactly canceled by the ghosts and we are left with the matter correlator

$$
2 i p\left\langle e^{i p X}, X\right\rangle=-\left.2 p \partial_{q}\left\langle e^{i p X}, e^{-i q X}\right\rangle\right|_{q=0}=2 p \delta^{\prime}(p)=-2 \delta(p),
$$

in agreement with (4.16). All the other coefficient calculations that we performed are not affected by this subtlety, since there the zero mode cancels out.

\section{Other marginal deformations}

The solution that we constructed is for the marginal deformation related to the $\partial X$ operator. However, we can generalize to other deformations with the same OPE, such as the $\cos (X)$ deformation. To realize how should the generalization look like we recall the logic of our construction. To find the $\partial X$ solution we introduced a primitive for $\partial X$, including a zero mode $x_{0}$, such that $Q X=c \partial X$. This enlargement of the Hilbert space allowed us to treat closed, non-exact states as if they were exact. Next we required that the solution does not depend on this zero mode, i.e., that it is well defined in the small Hilbert space. To that end we had to add counter terms of the form of powers of the primitive.

All that works exactly the same for $\cos (X)$. We first define the primitive

$$
\Xi(z)=\Xi_{0}+\int_{0}^{z} \cos (X(\tilde{z})) d \tilde{z}
$$

The form of the solution is the same as before, and the $\Xi_{0}^{n}$ terms drop out for same reasons. Since the result is $\Xi_{0}$ independent, it is given just by integrals of the $\cos (X)$ operator. Interestingly, in this representation, which can also be applied to the $\partial X$ case, there are $n-1$ integrals at order $n$, just like in the solutions [31, 30]. However, while there the integrals represent changing the size of a wedge state, here the wedge state is fixed and the integrations are over the positions of the insertions.

This analysis is, however, too naive. The problem is related to the issue of normal ordering. While the OPE's $\partial X \partial X$ and $\cos (X) \cos (X)$ have the same singular structure, they have different finite contributions, which alters the form of the solution. A possible resolution is to work with the $\cos (X)$ operator using a different normal ordering scheme, related to the modes in the expansion of its primitive. However, there are some subtleties in this route as well. We believe that these subtleties are of a technical nature, especially in light of the fact that the operators $\partial X$ and $\cos (X)$ are related by an $\mathrm{SU}(2)$ transformation and have the same form of commutation relation with $Q$. 
We would face similar problems in generalizing the construction to deformations such as $e^{X_{0}}$, whose $\mathrm{OPE}$ is regular. Again, it seems that defining a primitive and modifying the normal ordering scheme should be enough for defining a solution. We hope to return to these issues in the near future.

Our construction should work only for deformations which are exactly marginal. At first instance it may seem that we could generalize the construction also for vertex operators whose OPE behaves like

$$
V V \sim z^{-2}+z^{-1} V
$$

The caveat here is that the structure functions associated with normal ordering $V$ are $V$ dependent. Therefore, the proof below (2.17) regarding the regularity of the normal ordered expression, does not generalize to this case.

The one case where our construction can be directly generalized is $\partial X_{+}$. Inspecting (2.10) for this case shows that (2.11) is replaced in this case by

$$
\left[Q, X_{+}^{n}\right]=n c \partial X_{+} X_{+}^{n-1} .
$$

The form of the solution is the same, except that there are no $\partial c$ terms. Also, the terms that arose from normal ordering are now absent. This leads to a simplification in the evaluation of coefficients. In particular, for the tachyon and the light-cone photon we get

$$
A_{n}=0 \quad \forall n>1, \quad T_{n}=0 \quad \forall n .
$$

It would be interesting to compare this solution to the recently found one [32].

\section{Comparing with former solutions}

In this section we comment on the relation of our construction to that of Kiermaier, Okawa, Rastelli and Zwiebach and of Schnabl [30, 31]. The construction of these authors starts with any BRST-closed state in the Schnabl gauge

$$
Q \psi_{1}=0 \quad \mathcal{B}_{0} \psi_{1}=0
$$

Alternatively, one could work in the Siegel gauge or any other gauge, but the choice of Schnabl's gauge simplifies later calculations. A one-parameter family of solutions can be defined through

$$
\Psi=\sum_{n=1}^{\infty} \lambda^{n} \psi_{n}
$$

with

$$
\psi_{n} \equiv-\frac{\mathcal{B}_{0}}{\mathcal{L}_{0}} \sum_{k=1}^{n-1} \psi_{k} \psi_{n-k} .
$$

This solution might be ill defined because the $1 / \mathcal{L}_{0}$ operation is not a priori well defined, as $\mathcal{L}_{0}$ has a nontrivial kernel. Consequently, $\mathcal{B}_{0} / \mathcal{L}_{0}$ is only well-defined up to an addition of an arbitrary BRST-closed state. In fact, in the construction of [30, 31] adding such a term in some cases becomes necessary in order to cancel singularities. 
Adding BRST-closed states at each order is equivalent to adding them all at the first stage, provided that an explicit $\lambda$ dependence of $\psi_{n}$ is allowed. That is, given

$$
\tilde{\psi}_{n} \equiv-\frac{\mathcal{B}_{0}}{\mathcal{L}_{0}} \sum_{k=1}^{n-1} \tilde{\psi}_{k} \tilde{\psi}_{n-k}+\chi_{n}
$$

where $\chi_{n}$ are closed and $\tilde{\psi}_{n}$ are $\lambda$-independent, we obtain a solution $\tilde{\Psi}$ which equals the state $\Psi$ built from $\psi_{n}(\lambda)$, where

$$
\psi_{n>1}=-\frac{\mathcal{B}_{0}}{\mathcal{L}_{0}} \sum_{k=1}^{n-1} \psi_{k} \psi_{n-k}, \quad \psi_{1}=\sum_{n=1}^{\infty} \lambda^{n-1} \chi_{n} .
$$

This should be clear, since it simply amounts to a $\lambda$ expansion. A rigorous proof is given in appendix A.

The identity for $\psi_{1}$ in (6.5) expresses exactly the way two solutions that are identical at lowest order (i.e., have identical $\chi_{1}$ ) may differ at higher levels. Namely, we are free to take $\chi_{n>1}$ equal to any BRST-closed state. Adding to $\chi_{n}$ a term proportional to $\chi_{1}$ is equivalent to a redefinition of the deformation parameter as in (3.14). Adding to $\chi_{n}$ another BRST-closed state (corresponding to a linear combination of other linearized marginal deformations) leads, however, to a physically inequivalent solution. Only the addition of a BRST-exact state leads to a solution that is gauge equivalent to the original one. It is useful to note at this point that fixing the gauge only removes the ambiguity of adding a BRST-exact state, not of a more general, BRST-closed one. However, the $\mathcal{B}_{0}$ operator does not fix the gauge completely. Indeed, a pure gauge state satisfying the $\mathcal{B}_{0}$ gauge is the basis of Schnabl's solution.

In light of these considerations it is interesting to try and compare our solution to the solutions of [30, 31]. The latter obey the Schnabl gauge by construction. While this gauge choice breaks down when counter terms are added, it is true for the regular solutions. Our solutions on the other hand do not obey the $\mathcal{B}_{0}$ gauge. The general form of our solution is $\hat{U}_{n+1}$ acting on a combination of $c$ and $\partial c$ insertions at various points acting on matter insertions. The commutation relations

$$
\mathcal{B}_{0} \hat{U}_{n}=\hat{U}_{n}\left(\frac{4-n}{2} \mathcal{B}_{0}+\frac{2-n}{2} \mathcal{B}_{0}^{\dagger}\right), \quad\left[\mathcal{B}_{0}, c(z)\right]=z,
$$

imply that already $\phi_{2}$ does not obey the gauge condition even before counter terms are added, and adding them cannot alter this result.

Consequently, our solution and those of [30, 31] are in different gauges. Nevertheless, both solutions are built only by using the original linearized marginal solution $\psi_{1}$ and string field products thereof, and don't involve other linearized marginal solutions. For this reason we expect that these solutions are gauge equivalent, possibly after a $\lambda$ reparametrization.

\section{Conclusions}

We have presented the first explicit analytic solution corresponding to the photon marginal deformation $\partial X$. Our method leads, at first instance, to a non-normalizable dependence 
of the solution on the center of mass coordinate. Key part of our construction is that it is carefully tuned in order to cancel this dependence, leading to a solution in the physical Hilbert space. For a compact spatial direction, this deformation corresponds to giving a vev to the Wilson loop. The center of mass coordinate $x_{0}$ should be regarded in this context as a formal extension of the algebra.

One issue that remains open is the determination of the radius of convergence of the series defining the solution. Examination of the lowest-order coefficients for the tachyon state and the photon state suggests that this radius of convergence is finite. Another related issue is to find the functional relation between $\lambda_{\mathrm{CFT}}$ and $\lambda_{\mathrm{SFT}}$ used here.

While we presented explicitly the photon marginal deformation, is should also be possible to use our method for other operators with the same OPE, such as $\cos (X)$ or $\cosh \left(X^{0}\right)$. It would be interesting to identify the periodicity with respect to $\lambda_{\mathrm{CFT}}$ in terms of $\lambda_{\mathrm{SFT}}$. Also, since this marginal deformation connects the perturbative and the true vacua, it should give a new representation of the true vacuum and it may even be possible to explore the physics around it. Our method is particularly advantageous for operators that have a singular OPE in the coincidence limit, as it avoids corresponding singularities in the solution. However, it is also possible to use it for describing marginal deformations with regular OPE.

An interesting extension of our method would be to try and construct space-time dependent solutions, with a nontrivial but normalizable dependence on the center of mass coordinate. Another exciting possibility would be to use it in the context of supersymmetric string field theory. This may prove to be quite natural in light of the recent findings of 32,33$]$.

\section{Acknowledgments}

We benefited much from discussions with Nathan Berkovits, Ted Erler, Stefan Fredenhagen, Sanny Itzhaki, Yuji Okawa, Yaron Oz, Martin Schnabl, Ashoke Sen, Shigenori Seki and Stefan Theisen. E. F. and M. K. would like to thank the organizers of the HRI string workshop and of the ISM06 for providing stimulating research environments in enjoyable atmosphere and to Tel-Aviv University for hospitality. R. P. thanks the Albert-EinsteinInstitute for hospitality and financial support during his visits. The work of M. K. is supported by a Minerva fellowship. The work of E. F. is supported by the DIP foundation. The work of R. P. is supported by the Portuguese Fundação para a Ciência e a Tecnologia.

\section{A. Proving the equivalence of $(6.4)$ and $(6.5)$}

To prove that adding BRST-closed states order by order is equivalent to adding them all at the first stage, we expand

$$
\psi_{n}=\sum_{k=0}^{\infty} \psi_{n, k} \lambda^{k} .
$$


By expanding (6.5) with respect to $\lambda$ we get that these states obey the recursion relation

$$
\psi_{n>1, k}=-\frac{\mathcal{B}_{0}}{\mathcal{L}_{0}} \sum_{j=1}^{n-1} \sum_{l=0}^{k} \psi_{j, l} \psi_{n-j, k-l} .
$$

We now define

$$
\tilde{\psi}_{n} \equiv \sum_{m=1}^{n} \psi_{m, n-m}
$$

What we have to prove is that these states obey the recursion relation (6.4). For $n=1$ this hold trivially. For $n>1$, we plug (A.2) into (A.3) and get

$$
\tilde{\psi}_{n}=-\frac{\mathcal{B}_{0}}{\mathcal{L}_{0}}\left[\sum_{m=2}^{n} \sum_{j=1}^{m-1} \sum_{l=0}^{n-m} \psi_{j, l} \psi_{m-j, n-m-l}\right]+\chi_{n},
$$

where we separated the contribution from $m=1$. Comparing to (6.4), we see that what we have to prove is that the expression in the square brackets equals $\sum_{k=1}^{n-1} \tilde{\psi}_{k} \tilde{\psi}_{n-k}$. Note that nowhere did we use the equation of motion or any property of the operator in front of the square brackets other than being linear and well defined. Neither did we use the closeness of the $\chi_{n}$ 's. Now write this expression as

$$
\sum_{j=1}^{n-1} \sum_{r=1}^{n-j} \sum_{l=0}^{n-j-r} \psi_{j, l} \psi_{r, n-j-r-l}=\sum_{j=1}^{n-1} \sum_{k=j}^{n-1} \sum_{r=1}^{n-k} \psi_{j, k-j} \psi_{r, n-k-r}=\sum_{k=1}^{n-1} \tilde{\psi}_{k} \tilde{\psi}_{n-k},
$$

where we first interchanged the order of the first and second sums and defined $r=m-j$. Then, we defined $k=l+j$ and interchanged the second and third sums. Finally, we interchanged the order of the first two sums and used the definition (A.3). This completes the proof.

\section{References}

[1] M. Schnabl, Analytic solution for tachyon condensation in open string field theory, Adv. Theor. Math. Phys. 10 (2006) 433 hep-th/0511286.

[2] Y. Okawa, Comments on Schnabl's analytic solution for tachyon condensation in Witten's open string field theory, JHEP 04 (2006) 055 hep-th/0603159.

[3] E. Fuchs and M. Kroyter, On the validity of the solution of string field theory, JHEP 05 (2006) 006 hep-th/0603195.

[4] E. Fuchs and M. Kroyter, Schnabl's $\mathcal{L}_{0}$ operator in the continuous basis, JHEP 10 (2006) 067 hep-th/0605254.

[5] L. Rastelli and B. Zwiebach, Solving open string field theory with special projectors, hep-th/0606131.

[6] I. Ellwood and M. Schnabl, Proof of vanishing cohomology at the tachyon vacuum, JHEP 02 (2007) 096 hep-th/0606142. 
[7] H. Fuji, S. Nakayama and H. Suzuki, Open string amplitudes in various gauges, JHEP 01 (2007) 011 hep-th/0609047.

[8] E. Fuchs and M. Kroyter, Universal regularization for string field theory, JHEP 02 (2007) 038 hep-th/0610298.

[9] C. Imbimbo, The spectrum of open string field theory at the stable tachyonic vacuum, Nucl. Phys. B 770 (2007) 155 hep-th/0611343.

[10] T. Erler, Split string formalism and the closed string vacuum, JHEP 05 (2007) 083 hep-th/0611200.

[11] T. Erler, Split string formalism and the closed string vacuum. II, JHEP 05 (2007) 084 hep-th/0612050.

[12] E. Witten, Noncommutative geometry and string field theory, Nucl. Phys. B 268 (1986) 253.

[13] A. Sen, Descent relations among bosonic D-branes, Int. J. Mod. Phys. A 14 (1999) 4061 hep-th/9902105.

[14] A. Sen, Universality of the tachyon potential, JHEP 12 (1999) 027 hep-th/9911116.

[15] Y. Okawa, L. Rastelli and B. Zwiebach, Analytic solutions for tachyon condensation with general projectors, hep-th/0611110.

[16] A. Sen, On the background independence of string field theory, Nucl. Phys. B 345 (1990) 551.

[17] A. Sen, On the background independence of string field theory. 2. analysis of on-shell s matrix elements, Nucl. Phys. B 347 (1990) 270.

[18] A. Sen, On the background independence of string field theory. 3. Explicit field redefinitions, Nucl. Phys. B 391 (1993) 550 hep-th/9201041.

[19] B. Zwiebach, Closed string field theory: quantum action and the B-V master equation, Nucl. Phys. B 390 (1993) 33 hep-th/9206084.

[20] A. Sen and B. Zwiebach, Large marginal deformations in string field theory, JHEP 10 (2000) 009 hep-th/0007153.

[21] B. Zwiebach, A solvable toy model for tachyon condensation in string field theory, JHEP 09 (2000) 028 hep-th/0008227.

[22] A. Iqbal and A. Naqvi, On marginal deformations in superstring field theory, JHEP 01 (2001) 040 hep-th/0008127.

[23] T. Takahashi and S. Tanimoto, Marginal and scalar solutions in cubic open string field theory, JHEP 03 (2002) 033 hep-th/0202133.

[24] J. Kluson, Marginal deformations in the open bosonic string field theory for N D0-branes, Class. and Quant. Grav. 20 (2003) 827 hep-th/0203089.

[25] J. Kluson, Exact solutions in SFT and marginal deformation in BCFT, JHEP 12 (2003) 050 hep-th/0303199.

[26] F. Katsumata, T. Takahashi and S. Zeze, Marginal deformations and closed string couplings in open string field theory, JHEP 11 (2004) 050 hep-th/0409249.

[27] A. Sen, Energy momentum tensor and marginal deformations in open string field theory, JHEP 08 (2004) 034 hep-th/0403200. 
[28] H.-T. Yang and B. Zwiebach, Testing closed string field theory with marginal fields, JHEP 06 (2005) 038 hep-th/0501142.

[29] I. Kishimoto and T. Takahashi, Marginal deformations and classical solutions in open superstring field theory, JHEP 11 (2005) 051 hep-th/0506240.

[30] M. Kiermaier, Y. Okawa, L. Rastelli and B. Zwiebach, Analytic solutions for marginal deformations in open string field theory, hep-th/0701249.

[31] M. Schnabl, Comments on marginal deformations in open string field theory, hep-th/0701248.

[32] T. Erler, Marginal solutions for the superstring, JHEP 07 (2007) 050 arXiv:0704.0930.

[33] Y. Okawa, Analytic solutions for marginal deformations in open superstring field theory, arXiv:0704.0936.

[34] N. Berkovits, Super-Poincaré invariant superstring field theory, Nucl. Phys. B 450 (1995) 90 hep-th/9503099.

[35] L. Rastelli and B. Zwiebach, Tachyon potentials, star products and universality, JHEP 09 (2001) 038 hep-th/0006240.

[36] K. Furuuchi and K. Okuyama, Comma vertex and string field algebra, JHEP 09 (2001) 035 hep-th/0107101.

[37] L. Rastelli, A. Sen and B. Zwiebach, Boundary CFT construction of D-branes in vacuum string field theory, JHEP 11 (2001) 045 hep-th/0105168].

[38] M. Schnabl, Wedge states in string field theory, JHEP 01 (2003) 004 hep-th/0201095. 